\title{
Equilibrium, Regular Polygons, and Coulomb-Type Dynamics in Different Dimensions
}

\author{
W. I. Skrypnik \\ Institute of Mathematics, Tereshchenkivska 3, Kyiv-4 01004, Ukraine \\ Correspondence should be addressed to W. I. Skrypnik; volodymyr_skrypnyk@ukr.net
}

Received 20 December 2020; Revised 9 February 2021; Accepted 12 February 2021; Published 29 March 2021

Academic Editor: Ivan Giorgio

Copyright (c) 2021 W. I. Skrypnik. This is an open access article distributed under the Creative Commons Attribution License, which permits unrestricted use, distribution, and reproduction in any medium, provided the original work is properly cited.

\begin{abstract}
The equation of motion in $\mathbb{R}^{d}$ of $n$ generalized point charges interacting via the $s$-dimensional Coulomb potential, which contains for $d=2$ a constant magnetic field, is considered. Planar exact solutions of the equation are found if either negative $n-1>2$ charges and their masses are equal or $n=3$ and the charges are different. They describe a motion of negative charges along identical orbits around the positive immobile charge at the origin in such a way that their coordinates coincide with vertices of regular polygons centered at the origin. Bounded solutions converging to an equilibrium in the infinite time for the considered equation without a magnetic field are also obtained. A condition permitting the existence of such solutions is proposed.
\end{abstract}

\section{Introduction}

In this paper, we find solutions of the equation of motion in $\mathbb{R}^{d}$ of $n$ generalized point charges (or simply charges) $e_{j} \epsilon$ $\mathbb{R}, j=1, \cdots n$ with masses $m_{j}>0$ interacting via the pair $s$ -dimensional Coulomb potential, which is proportional to the fundamental solutions of the $s$-dimensional Laplacian [1]. In the case $d=2$, the equation also contains a constant magnetic field $h_{c}$ directed along the perpendicular to the plane containing the charges. The charge coordinates $x_{j} \in$ $\mathbb{R}^{d}$ obey the following equation of motion:

$m_{j} \frac{d^{2} x_{j}}{d t^{2}}=-\frac{\partial U\left(x_{(n)}\right)}{\partial x_{j}}+\delta_{d, 2} e_{j} \dot{x}_{j} \times h_{c}, \quad j=1, \cdots, n>2, \quad(1)$ where

$$
\begin{gathered}
U\left(x_{(n)}\right)=\sum_{1 \leq j<k \leq n} e_{j} e_{k} \varphi\left(x_{j}-x_{k}\right), \\
\varphi(x)=\left(1-\delta_{2, s}\right)|x|^{2-s}+\delta_{2, s} \ln |x|, \\
2 \leq s \in \mathbb{Z}^{+}, \\
x_{(n)}=\left(x_{1}, \cdots, x_{n}\right) \in \mathbb{R}^{d n}, \\
|x|^{2}=\left(x^{1}\right)^{2}+\cdots+\left(x^{d}\right)^{2} .
\end{gathered}
$$

$\dot{x}=d x_{j} / d t$ and the symbol $\times$ determines the skewsymmetric product. For $d=2$, one has $\left(\dot{x} \times h_{c}\right)^{1}=h \dot{x}^{2}$, $\left(\dot{x} \times h_{c}\right)^{2}=-h \dot{x}^{1}, h \in \mathbb{R}$, and $\delta_{d, 2}$ is the Kronecker symbol. The systems (1) and (2) without a magnetic field and their dynamics in the general case, case $s=3$, and case $d=3, s=3$ we call $(d, s)$-Coulomb, $d$-dimensional Coulomb, and Coulomb, respectively. 
Equation (1) is rewritten as follows:

$$
\begin{aligned}
m_{j} \frac{d^{2} x_{j}}{d t^{2}} & =g \sum_{k=1, k \neq j}^{n} e_{j} e_{k} \frac{x_{j}-x_{k}}{\left|x_{j}-x_{k}\right|^{s}}+\delta_{d, 2} e_{j} \dot{x}_{j} \times h_{c}, \quad j=1, \cdots, n, g \\
& =(s-2)\left(1-\delta_{2, s}\right)-\delta_{2, s} .
\end{aligned}
$$

The most important among the systems are those that describe the electrodynamics, that is, $d=1,2,3, s=2,3$, and the sequence of $e_{j}, j=1, \cdots, n$ contains numbers with different signs for them. We shall consider the systems with $n-1$ equal negative charges $-e_{0}$ and a positive charge $e_{n}$.

The profound result concerning the collision set for the dynamics determined by (3) without the magnetic field is obtained in [2] (see Remark 13).

In this paper, we find the regular polygon equilibrium, exact regular polygon solutions for $d=2$, and bounded solutions converging to the equilibrium in the infinite time limit. The regular polygon equilibrium is created by the system of $n-1$ equal negative charges $e_{0}$ located at all $n-1$ vertices of a regular polygon centered at the origin which is occupied by the central positive charge $e_{n}=e_{n}^{0}$. It is given in Theorem 7 and (32) showing that $e_{n}^{0}=2^{-1} e_{0}(n-2)$ for $s=2$ and $e_{3}^{0}=$ $2^{1-s} e_{0}$. For $n=3$, the regular polygon is represented by two opposite points on a circle.

This equilibrium allows one to find easily the exact planar regular polygon solutions. They describe an identical motion of every negative charge around the origin where the positive charge is immobile if $g\left(e_{n}-e_{n}^{0}\right)>0$. This condition induces a Keplerian-type motion of the negative charges and the Keplerian motion of them for the Coulomb systems $(s=3)$. Besides, the negative charges are tied to vertices of regular polygons centered at the origin all the time. In the simplest case, the negative charges rotate around the positive immobile charge with the same frequency. This is described by the following solution $x_{k}(t) \in \mathbb{C}$ of the equation of motion for negative charges (we consider $\mathbb{R}^{2}$ as $\mathbb{C}$ )

$$
x_{k}(t)=z_{k} e^{i u(t)} r(t), \quad r(t)>0,
$$

where $z_{k}, u(t), r(t)$ satisfy the first, second, and third structure equations, respectively, the first of which does not depend on time. Here, $z_{k}$ are coordinates of the regular polygon vertices and the equation for $u$ means conservation of the kinetic moment of a two-dimensional mechanical system. The third structure equation is its equation of motion for the radial variable. Earlier, we obtained the same result for the Coulomb systems with the zero magnetic field [3].

For the system of the three charges, we prove the following theorem.

Theorem 1. Let (3) with $h_{c}=0, n=3$ determine the dynamics of the three point charges $e_{1}=-e_{0}, e_{2}=-e_{0}, e_{3}=2^{1-s} e_{0}>0$ with masses $m_{j}, j=1,2,3$. Then, it possesses a bounded at positive time solution which is a real analytic function in a neigh- borhood of the origin in one real parameter for $s>2$ and $d-1$ real parameters for $s=2, d>1$ such that $\left\|x-x^{0}\right\|_{\lambda}<\infty$, $\|\dot{x}\|_{\lambda}<\infty$, where $x^{0}$ is the equilibrium $x_{1}^{01}=-a, x_{2}^{01}=a>0$, $x_{3}^{01}=0, x_{j}^{0 \alpha}=0,1<\alpha \leq d, 1 \leq j \leq 3$, and $\lambda<\lambda_{0}, \lambda_{0}^{2}=e_{0}^{2}|g|(s$ $-1)(2 a)^{-s}\left(m_{1}^{-1}+m_{2}^{-1}+4 m_{3}^{-1}\right), \dot{x}$ is the velocity, and

$$
\|x\|_{\lambda}=\sup _{t \geq 0} \max _{j \in(1, \cdots, n)} e^{\lambda t}\left|x_{j}(t)\right|_{0}, \quad\left|x_{j}(t)\right|_{0}=\max _{1 \leq \alpha \leq d}\left|x_{j}^{\alpha}(t)\right| .
$$

We were able to prove this theorem since we found all eigenvalues of the matrix $\left(M^{-1} U^{0}\right)_{j, \alpha ; j, \alpha}$, where $M$ is the diagonal matrix in $\mathbb{R}^{d n}$ with elements $M_{j, \alpha}=m_{j}, 1 \leq j \leq n$, and 1 $\leq \alpha \leq d$ and $U_{j, \alpha<l, \beta}^{0}$ is the matrix of the second partial derivatives $U_{j, \alpha ; j, \beta}$ of the potential energy at the regular polygon equilibrium

$$
U_{j, \alpha ; l, \beta}=\frac{\partial^{2} U\left(x_{(n)}\right)}{\partial x_{j}^{\alpha} \partial x_{l}^{\beta}} .
$$

We prove also the following theorem.

Theorem 2. Let $U$ in (2) possess an equilibrium $x^{0}$ and $T r$ $M^{-1} U^{0} \leq 0$, where

$$
\operatorname{Tr}^{-1} U^{0}=\sum_{j=1}^{n} m_{j}^{-1} \sum_{\alpha=1}^{d} U_{j, \alpha ; j, \alpha}^{0} .
$$

Then, there exists an integer $p, 1 \leq p<n d$, a positive number $\lambda$, and a bounded at positive time solution $x(t)$ of the equation of motion (3) with $h_{c}=0$ depending on $p$ real parameters, which is real analytic function in them in a neighborhood of the origin, such that $\left\|x-x^{0}\right\|_{\lambda}<\infty,\|\dot{x}\|_{\lambda}<\infty$.

If $s=d$, then $\operatorname{Tr} M^{-1} U^{0}=0$ for an arbitrary equilibrium for our systems without a magnetic field since

$$
\begin{gathered}
\sum_{\alpha=1}^{d} U_{j, \alpha ; j, \alpha}=0, \\
U_{j, \alpha ; j, \beta}=g e_{j} \sum_{k=1, k \neq j}^{n} e_{k}\left[-\frac{\delta_{\alpha, \beta}}{\left|x_{j}-x_{k}\right|^{s}}+s \frac{\left(x_{j}^{\alpha}-x_{k}^{\alpha}\right)\left(x_{j}^{\beta}-x_{k}^{\beta}\right)}{\left|x_{j}-x_{k}\right|^{s+2}}\right] .
\end{gathered}
$$

In our calculations of partial derivatives of the potential energy, we use the vector-valued equalities 


$$
\begin{aligned}
\frac{\partial}{\partial x_{1}}\left|x_{1}-x_{2}\right|^{-k} & =-k \frac{x_{1}-x_{2}}{\left|x_{1}-x_{2}\right|^{k+2}}, \quad x_{j} \in \mathbb{R}^{d}, \\
\frac{\partial}{\partial x_{1}} \ln f\left(x_{1}\right) & =f^{-1}\left(x_{1}\right) \frac{\partial}{\partial x_{1}} f\left(x_{1}\right), \\
\frac{\partial}{\partial x_{1}} \ln \left|x_{1}-x_{2}\right| & =\frac{x_{1}-x_{2}}{\left|x_{1}-x_{2}\right|^{2}}, \\
\frac{\partial}{\partial x_{j}} U\left(x_{(n)}\right) & =-g e_{j} \sum_{k=1, k \neq j}^{n} e_{k} \frac{x_{j}-x_{k}}{\left|x_{j}-x_{k}\right|^{s}} .
\end{aligned}
$$

Theorem 2 implies the following result.

Theorem 3. Let $U$ in (2) possess an equilibrium $x^{0}$ and $s=d$. Then, the conclusion of Theorem 2 is true.

In order to estimate $\operatorname{Tr} M^{-1} U^{0}$ in the case $s \neq d$, one has to consider a particular equilibrium. We shall choose in the next theorem the regular polygon equilibrium for $n$ charges (see Corollary 8).

Theorem 4. For the regular polygon equilibrium for $n-1$ equal negative charges $-e_{0}$ and central positive charge $e_{n}^{0}$, the following equality is true:

$\operatorname{Tr} M^{-1} U^{0}=\frac{e_{0}^{2}}{a^{s}} g(d-s)\left[m^{\prime}\left(e_{0}^{-1} e_{n}^{0}-e_{n, s}\right)+m_{n}^{-1}(n-1) e_{0}^{-1} e_{n}^{0}\right]$,

where $m^{\prime}=\sum_{l=1}^{n-1} m_{l}^{-1}$,

$e_{n, s}=2^{-s / 2} \sum_{k=1}^{n-2}\left(1-\cos \frac{2 \pi k}{n-1}\right)^{-s / 2}=2^{-s} \sum_{k=1}^{n-2} \sin ^{-s} \frac{\pi k}{n-1}, \quad e_{n, s-2}=2 e_{0}^{-1} e_{n}^{0}$.

An easy calculation at the end of the fourth section shows that $\operatorname{Tr} M^{-1} U^{0}<0$ for $s>d$ if $n=3,4,5$ (see Remark 14).

Note that the condition of the neutrality $e_{n}=e_{0}(n-1)$ is impossible for the regular polygon equilibrium for $s=2$.

The conclusion of Theorem 3 is true for the 3 dimensional Coulomb systems of $n+2$ and $n+1$ charges $n$ -1 of which are equal considered in $[4,5]$, respectively. In their equilibria, the equal negative charges are located at all the vertices of a regular polygon centered at the origin while the rest nonplanar charges are located at the perpendicular crossing the origin. In [4], a positive charge occupies the origin and the nonplanar charges are equal and may have different signs. In [5], the nonplanar charges are equal and positive. The solutions of the equation of motion are found in these papers provided the masses of the equal negative charges are equal. in [6].

Theorem 2 relies on the following basic theorem proven

Theorem 5. Let $U^{0}$ be the symmetric matrix of second-order partial derivatives of $U$ at an equilibrium $x^{0}$ Let also $U$ be a real analytic function in a neighborhood of $x^{0}$ and the matrix $M^{-1} U^{0}$ have $p$ negative eigenvalues $\sigma_{j}, j=1, \cdots, p$. Then, (1) with $h_{c}=0$ possesses a bounded at positive time solution $x_{j}(t)$, $1 \leq j \leq n$ depending on $p$ real parameters which is a real analytic function in them in a neighborhood of the origin and $\left\|x-x^{0}\right\|_{\lambda}<\infty,\|\dot{x}\|_{\lambda}<\infty$, where $\dot{x}$ is the velocity and

$$
\|x\|_{\lambda}=\sup _{t \geq 0} \max _{j \in(1, \cdots, n)} e^{\lambda t}\left|x_{j}(t)\right|, \quad \lambda<\lambda_{0}=\min _{j=1, \cdots, p} \sqrt{-\sigma_{j}} .
$$

For $d>1$, the pair potentials and potential energies in (2) are real analytic functions in a neighborhood of $a_{j} \in \mathbb{R}^{d}, 1$ $\leq j \leq n, a_{j} \neq a_{k}$, i.e., a set where coordinates do not coincide ( $a_{j}$ may correspond to an equilibrium). This follows from the fact that the Taylor power expansion for $x^{-s}$ converges absolutely and uniformly in a neighborhood of a point from $\mathbb{R}^{+}$. In the one-dimensional case, the chosen order of charges is preserved by the dynamics close to the equilibrium and one can change $\left|x_{j}-x_{k}\right|^{2-s}, \ln |x|$ by the real analytic functions $\left(x_{j}-x_{k}\right)^{2-s}, \ln \left(x_{j}-x_{k}\right), x_{j}>x_{k}$. So one can apply Theorem 5 to (1) and (2).

Theorem 1 follows from Theorem 12 and Theorem 5 . From the equality $\sqrt{M} M^{-1} U^{0}(\sqrt{M})^{-1}=(\sqrt{M})^{-1} U^{0}(\sqrt{M})^{-1}$ , it follows that the matrix $M^{-1} U^{0}$ has the same spectrum as the symmetric matrix $(\sqrt{M})^{-1} U^{0}(\sqrt{M})^{-1}$ and is similar to the diagonal matrix with real elements. This leads to the important conclusion: if $\operatorname{Tr} M^{-1} U^{0} \leq 0$, then there exists at least one negative eigenvalue of $M^{-1} U^{0}$ since the trace coincides with the sum of the eigenvalues of the matrix. This and Theorem 5 prove Theorem 2 .

Our paper is organized as follows. In the second section, we introduce the three structure equations and explain in Proposition 6 how to construct with its help the exact solutions of (1) when there are equilibrium configurations in the form of regular polygons. Theorem 7 establishes their existence. In the third section, we consider the three charge cases and show that the exact solutions exist for which the charges are located at a common line provided the positive charge is greater than the absolute values of the rest two charges. In the fourth section, we prove Theorem 4 . Eigenvalues of $M^{-1} U^{0}$ for the system of charges from Theorem 1 are calculated in the fifth section. This result is formulated in Theorem 12 .

\section{Equilibrium and Exact Planar Dynamics}

Let $d=2$ and $x_{k}=x_{k}^{1}+i x_{k}^{2} \in \mathbb{C}$. Then, the analogue of the exact Lagrange solutions of the three-body problem [7] is determined by the equalities $x_{k}=z_{k} q(t)$ which give

$$
\left|x_{j}-x_{k}\right|=\left|z_{j}-z_{k}\right||q|
$$

From the equation of motion (3) with the contribution of magnetic field $-i e_{j} h \dot{x}_{j}$, it follows that 


$$
m_{j} z_{j} \frac{d^{2} q}{d t^{2}}=g q|q|^{-s} \sum_{k=1, k \neq j}^{n} e_{j} e_{k} \frac{z_{j}-z_{k}}{\left|z_{j}-z_{k}\right|^{s}}-i z_{j} e_{j} h \dot{q} .
$$

Sometimes, the $s$-dimensional Coulomb potential which leads to this equation is mentioned as the Riesz potential [8] if $s>2$ (see also [9]).

Let

$$
q(t)=e^{i u(t)} r(t), \quad r(t)>0
$$

and the following first structure equation hold

$$
-w^{2} m_{j} z_{j}=g \sum_{k=1, k \neq j}^{n} e_{j} e_{k} \frac{z_{j}-z_{k}}{\left|z_{j}-z_{k}\right|^{s}}, \quad w^{2}>0 .
$$

The first structure equation reduces the equation of motion to the following single equation:

$$
\frac{d^{2} q}{d t^{2}}=-w^{2} q|q|^{-s}-i b \dot{q}, \quad b=\frac{e_{0} h}{m} .
$$
Then,

Let $q=e^{i u(t)} r(t)$, where $u, r$ are real valued functions.

$$
\begin{gathered}
e^{-i u(t)} \frac{d^{2} q}{d t^{2}}=\frac{d^{2} r}{d t^{2}}+i\left(2 \frac{d r}{d t} \frac{d u}{d t}+\frac{d^{2} u}{d t^{2}} r\right)-\left(\frac{d u}{d t}\right)^{2} r \\
e^{-i u(t)} \dot{q}=i \dot{u} r+\dot{r} .
\end{gathered}
$$

The imaginary part of (17) multiplied by $e^{-i u}$ has to be zero

$$
2 \frac{d r}{d t} \frac{d u}{d t}+\frac{d^{2} u}{d t^{2}} r+b \frac{d r}{d t}=r^{-1} \frac{d}{d t}\left(r^{2} \frac{d u}{d t}+\frac{b}{2} r^{2}\right)=0
$$

As a result, the equation of motion yields the following second and third structure equations:

$$
\begin{gathered}
I=r^{2} \frac{d u}{d t}+r^{2} \frac{b}{2}=\eta \\
\frac{d^{2} r}{d t^{2}}-\left(\frac{d u}{d t}\right)^{2} r-b \frac{d u}{d t} r+w^{2} r^{1-s}=0
\end{gathered}
$$

where $\eta \in \mathbb{R}$ is a constant. For $b=0$, they look like

$$
\begin{gathered}
\frac{d u}{d t}=r^{-2} \eta \\
\frac{d^{2} r}{d t^{2}}-\eta^{2} r^{-3}+w^{2} r^{1-s}=0
\end{gathered}
$$

The third structure equation is the radial Kepler's equation for $s=3$. Note that if $b=0, r=1$, then the last equalities imply $u= \pm t w$. In the general case, if $r=1$, then from (17), it follows that

$$
\begin{gathered}
q(t)=e^{i w^{\prime} t} \\
w^{\prime 2}=w^{2}+b w^{\prime}, \quad w^{\prime}=\frac{b}{2} \pm \sqrt{\frac{b^{2}}{4}+w^{2}}
\end{gathered}
$$

Equation (17) for $q$ is the equation of motion of a twodimensional mechanical system in a constant magnetic field characterized by the kinetic moment $I$. Let us prove that it is defined also by

$$
I=q \times \dot{q}+\frac{b r^{2}}{2}
$$

and that it is an integral of motion. The skew-symmetric product is determined on $C^{2}$ as follows:

$$
\begin{aligned}
z & =x+i y, \\
q & =p+i v, \\
z \times q & =x v-y p, \\
e^{i u} x \times e^{i u} p & =(\cos u+i \sin u) x \times \cos u+i \sin u) p) \\
& =(\sin u) \cos u[i x \times p+x \times i p] \\
& =(\sin u) \cos u[-x p+x p]=0, \\
e^{i u} i y \times e^{i u} p & =(\cos u+i \sin u) i y \times \cos u+i \sin u) p) \\
& =\sin ^{2} u(y \times i p)+\left(\cos ^{2} u\right)(i y \times p) \\
& =-\left(\sin ^{2} u\right) y p-\left(\cos ^{2} u\right) y p=-y p .
\end{aligned}
$$

That is

$$
q \times \dot{q}=-e^{i u}(i u r r+\dot{r}) \times e^{i u} r=\dot{u} r^{2}
$$

As a result,

$$
\begin{aligned}
\dot{I} & =\dot{q} \times \dot{q}+q \times \ddot{q}+b r \dot{r}=b r \dot{r}+q \times\left[-w^{2} q|q|^{-s}-i b \dot{q}\right] \\
& =b r \dot{r}-w^{2}|q|^{-s}(q \times q)+b(i \ddot{q} \times q)=b r \dot{r}+b(i \dot{q} \times q) \\
& =b r \dot{r}-b \dot{u}\left(e^{i u} r \times e^{i u} r\right)+b\left(e^{i u} i \dot{r} \times e^{i u} r\right) \\
& =b r \dot{r}+b\left(e^{i u} i \dot{r} \times e^{i u} r\right)=0 .
\end{aligned}
$$

Now, we have to prove that solutions of the first structure equation exist. A crucial role in our proofs is played by the equality for the coordinates of the regular polygon vertices

$$
\sum_{k=1}^{n-1} z_{k}=0, \quad z_{k}=z^{k}, z=e^{i(2 \pi /(n-1))}
$$

which in its turn is a consequence of the equality

$$
z \sum_{k=1}^{n-1} z_{k}=\sum_{k=1}^{n-1} z_{k}
$$

Proposition 6. Let the equilibrium for $U$ in (2) be determined by the positive charge $e_{n}^{0}>0$ with the coordinate $z_{n}=0$ and negative charges $e_{j}^{0}=-e_{0}<0, j=1, \cdots, n-1$ with the equal 
mass $m$ be placed at all vertices $z_{j}$ of a regular polygon and $\mid$ $z_{n}-z_{j} \mid=a$ if $n>3$. Let also $z_{1}=-z_{2}=a$ if $n=3$. Then, the first structure equation is true if

$$
w^{2} a^{s} \frac{m}{e_{0}}=g\left(e_{n}-e_{n}^{0}\right)>0 .
$$

Proof. From $z_{n}=0$, it follows that for $j<n$ the right-hand side of the first structure equation looks like

$$
\begin{aligned}
\sum_{k=1, k \neq j}^{n} e_{j} e_{k} \frac{z_{j}-z_{k}}{\left|z_{j}-z_{k}\right|^{s}} & =\sum_{k=1, k \neq j}^{n} e_{j}^{0} e_{k}^{0} \frac{z_{j}-z_{k}}{\left|z_{j}-z_{k}\right|^{s}}-\left(e_{n}-e_{n}^{0}\right) e_{0} a^{-s} z_{j} \\
& =-\left(e_{n}-e_{n}^{0}\right) e_{0} a^{-s} z_{j} .
\end{aligned}
$$

From $z_{n}=0$ and (27), it follows that the first structure equation is true for $j=n$

$$
\sum_{k=1, k \neq j}^{n} e_{j} e_{k} \frac{z_{j}-z_{k}}{\left|z_{j}-z_{k}\right|^{s}}=-a^{-s} e_{0}^{2} \sum_{k=1}^{n-1} z_{k}=0
$$

The proposition is proved.

From (29), it follows that $e_{n}>e_{n}^{0}$ and $e_{n}<e_{n}^{0}$ for $s>2$ and $s=2$, respectively.

Theorem 7. Let the positive charge $e_{n}^{0}>0$ have the coordinate $z_{n}=0$, negative charges $e_{j}^{0}=-e_{0}<0, j=1, \cdots, n-1$ be placed at all vertices $z_{j}$ of a regular polygon and $\left|z_{n}-z_{j}\right|=a$ if $n>$ 3. Let also $z_{1}=-z_{2}=a$ if $n=3$. Then, such the configuration is an equilibrium for $U$ in (17) for $d=2$ if

$$
e_{n}^{0}=2^{-\frac{s}{2}} e_{0} \sum_{k=1}^{n-2}\left(1-\cos \frac{2 \pi k}{n-1}\right)^{(2-s) / 2}=2^{1-s} e_{0} \sum_{k=1}^{n-2} \sin ^{2-s} \frac{\pi k}{n-1}
$$

Proof. For $j=n$, the equilibrium condition is satisfied

$$
\sum_{k=1}^{n-1} e_{n}^{0} e_{k}^{0} \frac{z_{n}-z_{k}}{\left|z_{n}-z_{k}\right|^{s}}=e_{0} e_{n}^{0} a^{-s} \sum_{k=1}^{n-1} z_{k}=0
$$

For $j<n$, it yields

$$
\begin{aligned}
\sum_{k=1, k \neq j}^{n} e_{j}^{0} e_{k}^{0} \frac{z_{j}-z_{k}}{\left|z_{j}-z_{k}\right|^{s}} & =e_{0}^{2} \sum_{k=1, k \neq j}^{n-1} \frac{z_{j}-z_{k}}{\left|z_{j}-z_{k}\right|^{s}}-\frac{e_{0} e_{n}^{0}}{a^{s}} z_{j} \\
= & -z_{j} a^{-s} e_{0}\left(e_{n}^{0}-e_{0} \sum_{k=1, k \neq j}^{n-1} \frac{a^{s}}{\left|z_{j}-z_{k}\right|^{s}}\right) \\
& -e_{0}^{2} \sum_{k=1, k \neq j}^{n-1} \frac{z_{k}}{\left|z_{j}-z_{k}\right|^{s}}=0 .
\end{aligned}
$$

If one shows that

$$
\begin{aligned}
& \sum_{k=1, k \neq j}^{n-1} \frac{a^{s}}{\left|z_{j}-z_{k}\right|^{s}}=a_{n}, \\
& \sum_{k=1, k \neq j}^{n-1} \frac{a^{s} z_{k}}{\left|z_{j}-z_{k}\right|^{s}}=a_{n}^{\prime} z_{j},
\end{aligned}
$$

where

$$
\begin{gathered}
a_{n}=\sum_{k=1}^{n-2} \frac{1}{\left|1-z^{k}\right|^{s}}, \\
a_{n}^{\prime}=\sum_{k=1}^{n-2} \frac{z^{k}}{\left|1-z^{k}\right|^{s}}, \quad z=e^{i(2 \pi /(n-1))},
\end{gathered}
$$

then the following equality is proved:

$$
e_{n}^{0}=e_{0}\left(a_{n}-a_{n}^{\prime}\right)
$$

To prove these equalities, one has to prove for coordinates $z_{j}$ of the regular polygon such that $\left|z_{j}\right|=1$

$$
\begin{gathered}
\sum_{k=1, k \neq j}^{n-1} \frac{1}{\left|z_{j}-z_{k}\right|^{s}}=a_{n}, \\
\sum_{k=1, k \neq j}^{n-1} \frac{z_{k}}{\left|z_{j}-z_{k}\right|^{s}}=a_{n}^{\prime} z_{j} .
\end{gathered}
$$

To prove (39) ((38) is proved the same way), we have to take into account that $z_{k}=z^{k}, z=e^{i(2 \pi /(n-1))}, z^{-l}=z^{n-1-l}$

$$
\begin{aligned}
\sum_{k=1, k=j}^{n-1} \frac{z_{k}}{\left|z_{j}-z_{k}\right|^{s}} & =z_{j} \sum_{k=1, k=j}^{n-1} \frac{z^{k-j}}{\left|1-z^{k-j}\right|^{s}} \\
& =z_{j}\left(\sum_{k=1}^{j-1} \frac{z^{k-j}}{\left|1-z^{k-j}\right|^{s}}+\sum_{k=j+1}^{n-1} \frac{z^{k-j}}{\left|1-z^{k-j}\right|^{s}}\right) \\
& =z_{j}\left(\sum_{l=1}^{j-1} \frac{z^{-l}}{\left|1-z^{-l}\right|^{s}}+\sum_{l=1}^{n-j-1} \frac{z^{l}}{\left|1-z^{l}\right|^{s}}\right) \\
& =z_{j}\left(\sum_{l=1}^{j-1} \frac{z^{(n-l-1)}}{\left|1-z^{(n-l-1)}\right|^{s}}+\sum_{l=1}^{n-j-1} \frac{z^{l}}{\left|1-z^{l}\right|^{s}}\right) \\
& =z_{j} \sum_{l=1}^{n-2} \frac{z^{l}}{\left|1-z^{l}\right|^{s}} .
\end{aligned}
$$

Hence, the multiplier of $z_{j}$ does not depend on $j$ and it is real since 


$$
\begin{aligned}
\left(\sum_{l=1}^{n-2} \frac{z^{l}}{\left|1-z^{l}\right|^{s}}\right)^{*} & =\sum_{l=1}^{n-2} \frac{z^{-l}}{\left|1-z^{-l}\right|^{s}} \\
& =\sum_{l=1}^{n-2} \frac{z^{(n-l-1)}}{\left|1-z^{-(n-l-1)}\right|^{s}} \\
& =\sum_{l=1}^{n-2} \frac{z^{l}}{\left|1-z^{l}\right|^{s}} .
\end{aligned}
$$

Hence, (29) yields

$$
e_{n}^{0}=e_{0}\left(\sum_{k=1}^{n-2} \frac{1-\operatorname{Re} z^{k}}{\left|1-z^{k}\right|^{s}}\right)=2^{-\frac{s}{2}} e_{0} \sum_{k=1}^{n-2}\left(1-\cos \frac{2 \pi k}{n-1}\right)^{-(s-2) / 2}
$$

since $\operatorname{Re} z^{k}=\cos (2 \pi k /(n-1))$,

$$
\begin{aligned}
\left|1-z^{k}\right|^{2}= & 1-2 \cos \frac{2 \pi k}{n-1}+\cos ^{2} \frac{2 \pi k}{n-1} \\
& +\sin ^{2} \frac{2 \pi k}{n-1}=2\left(1-\cos \frac{2 \pi k}{n-1}\right) .
\end{aligned}
$$

But $1-\cos 2 x=2 \sin ^{2} x$. This concludes the proof.

Let $U$ correspond to the $(d, s)$-Coulomb system and

$$
\begin{aligned}
x_{k} & =\left(x_{k}^{\alpha}, \alpha=1, \cdots, d\right)=\left(g_{k}, x_{k}^{\prime}\right), g_{k} \in C, x_{k}^{\prime} \\
& =\left(x_{k}^{2}, \cdots, x_{k}^{d}\right) \in R^{d-2}, g_{k}^{1}=x_{k}^{1}=\operatorname{Re} g_{k}, g_{k}^{2}=x_{k}^{2}=\operatorname{Im} g_{k} .
\end{aligned}
$$

From

$$
\frac{\partial}{\partial x_{j}^{\alpha}} U\left(x_{(n)}\right)=-g e_{j} \sum_{k=1, k \neq j}^{n} e_{k} \frac{x_{j}^{\alpha}-x_{k}^{\alpha}}{\left|x_{j}-x_{k}\right|^{s}}, \quad \alpha=1, \cdots, d,
$$

it follows

$$
\frac{\partial}{\partial x_{j}^{\alpha}} U\left(x_{(n)}\right)=0, \quad \alpha>2, x_{j}^{\alpha}=0
$$

and the equilibrium is found from the equality

$$
\begin{gathered}
\frac{\partial}{\partial g_{j}} U\left(x_{(n)}\right)=\frac{\partial}{\partial g_{j}} U\left(g_{(n)}\right), \\
x_{j}^{\alpha}=0, \quad \alpha>2 .
\end{gathered}
$$

Hence, we proved the corollary with the help of Theorem 7.

Corollary 8. The regular polygon equilibrium determined in Theorem 7 exists for the $(d, s)$-Coulomb system in $\mathbb{R}^{d}$ in the plane $x^{\alpha}=0, \alpha=3, \cdots$, $d$ for $d \geq 2$.

\section{Three Nonequal Charges on a Plane}

In this section, we shall consider the first structure equation for three charges $e_{j}<0, j=1,2, e_{3}>0$. We will assume that their inertia center is immobile: $m_{1} z_{1}+m_{2} z_{2}+m_{3} z_{3}=0$. We treat $z_{j}$ as points of $\mathbb{R}^{2}$. Note that we assume that $e_{n}>$ $e_{n}^{0}$ for $s>2$ and $e_{n}<e_{n}^{0}$ for $s=2$.

Proposition 9. Solutions of the first structure equation for $n$ $=3$ do not coincide with the coordinates of vertices of a triangle.

Proof. It is possible to put the first charge at a coordinate axis. Let $z_{1}^{2}=0$. Then, the first structure equation for $z_{1}^{2}$ gives

$$
e_{2} z_{2}^{2}\left|z_{1}-z_{2}\right|^{-s}+e_{3} z_{3}^{2}\left|z_{1}-z_{3}\right|^{-s}=0
$$

Since the center of inertia is immobile, this equality results in

$$
z_{2}^{2}\left(\frac{\left|e_{2}\right|}{m_{2}}\left|z_{1}-z_{2}\right|^{-s}+\frac{e_{3}}{m_{3}}\left|z_{1}-z_{3}\right|^{-s}\right)=0
$$

That is, $z_{2}^{2}=0$ and $z_{3}^{2}=0$. This concludes the proof.

Proposition 10. If $e_{1}=e_{2}=-e_{0}, m_{1}=m_{2}=m_{0}, 2^{s-1} e_{3}>e_{0}$, then the rectilinear solution of the first structure equation for $n=3$ is given by $z_{j}^{2}=0, z_{3}^{1}=0,-z_{1}^{1}=z_{2}^{1}=2^{-1} a$ where

$$
w^{2} a^{s}=\frac{g 2^{s} e_{0}}{m_{0}}\left(e_{3}-2^{1-s} e_{0}\right)>0 .
$$

Proof. Let the charges are placed at a first coordinate axis: $z_{j}^{2}=0, q_{1}=z_{1}^{1}<q_{3}=z_{3}^{1}<q_{2}=z_{2}^{1}$ and $q_{2}-q_{1}=a, q_{3}-q_{1}=a$ $\rho, q_{2}-q_{3}=a \sigma, a>0$. Then, the first structure equation leads to

$$
\begin{gathered}
-e_{3}(\rho a)^{1-s}-e_{2} a^{1-s}=-\frac{m_{1}}{g e_{1}} w^{2} q_{1}, \\
e_{1}(\rho a)^{1-s}-e_{2}(\sigma a)^{1-s}=-\frac{m_{3}}{g e_{3}} w^{2} q_{3}, \\
e_{1} a^{1-s}+e_{3}(\sigma a)^{1-s}=-\frac{m_{2}}{g e_{2}} w^{2} q_{2} .
\end{gathered}
$$

Let $\rho=\sigma=1 / 2$, then the second equation gives $q_{3}=0$. The first and the third ones show that $-q_{1}=q_{2}=a / 2$ and $a$, $w$ satisfy the condition indicated in the formulation of this proposition. This concludes the proof.

Note that if one multiplies $a$ by 2 in Proposition 9, then $w$ in it and $w$ in Proposition 6 coincide since $e_{3}^{0}=2^{1-s} e_{0}$. If the conditions of the last proposition do not hold, that is, $e_{1} \neq$ $e_{2}$, then one can apply the following proposition.

Proposition 11. If $e_{3} \geq-e_{j}>0, j=1,2$, then a rectilinear solution of the first structure equation exists. 
Proof. Let us put $m_{*}=m_{1}+m_{2}+m_{3}$. From the equality $m_{1}$ $z_{1}+m_{2} z_{2}+m_{3} z_{3}=0$, it follows that

$$
m_{*} q_{1}+m_{3} \rho a+m_{2} a=0, m_{*} q_{2}-m_{3} \sigma a-m_{1} a=0
$$

Taking this into account, we see that the first and the third equalities look like $\left(q_{1}, q_{3}\right.$ are excluded)

$$
\begin{aligned}
& e_{3} \rho^{1-s}+e_{2}=-m_{*}^{-1} \frac{m_{1}}{g e_{1}} w^{2} a^{s}\left(m_{3} \rho+m_{2}\right), \\
& e_{3} \sigma^{1-s}+e_{1}=-m_{*}^{-1} \frac{m_{2}}{g e_{2}} w^{2} a^{s}\left(m_{3} \sigma+m_{1}\right) .
\end{aligned}
$$

Since $\sigma=1-\rho$,

$-m_{*}^{-1} w^{2} a^{s}=\frac{g e_{1}}{m_{1}} \frac{e_{3} \rho^{1-s}+e_{2}}{\left(m_{3} \rho+m_{2}\right)}=\frac{g e_{2}}{m_{2}} \frac{e_{3}(1-\rho)^{1-s}+e_{1}}{\left(m_{3}(1-\rho)+m_{1}\right)}, \quad 0<\rho<1$.

As a result in order to solve the first and third equations from the previous proposition, one has to show that $f=0$ has solutions in the interval $0<\rho<1$ such that $e_{3} \rho^{1-s}>\left|e_{2}\right|, e_{3}$ $(1-\rho)^{1-s}>\left|e_{1}\right|$ if $e_{1}, e_{2}<0, e_{3}>0$ and

$$
f(\rho)=\frac{e_{1}}{m_{1}} \frac{e_{3} \rho^{1-s}-\left|e_{2}\right|}{\left(m_{3} \rho+m_{2}\right)}-\frac{e_{2}}{m_{2}} \frac{e_{3}(1-\rho)^{1-s}-\left|e_{1}\right|}{\left(m_{3}(1-\rho)+m_{1}\right)} .
$$

If $e_{3} \geq-e_{j}>0, j=1,2$, then the equation $f=0$ has such the solutions since $f$ is a continuous function tending to $\infty, \infty$ at 0,1 , respectively. $q_{3}$ is found from the second structure equation. This concludes the proof.

\section{Equilibrium Character}

In this section, we calculate the trace

$$
\operatorname{Tr} M^{-1} U^{0}=\sum_{j=1}^{n} m_{j}^{-1} \sum_{\alpha=1}^{d} U_{j, \alpha ; j, \alpha}^{0}
$$

where $U_{j, \alpha: l, \beta}^{0}$ is the matrix of the second derivatives of the potential energy at the regular polygon equilibrium created by the system of $n-1$ equal negative charges located at all $n-1$ vertices of a regular polygon centered at the origin where the central positive charge $e_{n}^{0}$ is located.

We have

$$
U_{n, \alpha ; n, \beta}=\frac{\partial^{2} U\left(x_{(n)}\right)}{\partial x_{n}^{\alpha} \partial x_{n}^{\beta}}=-g e_{0} e_{n}^{0} \sum_{k=1}^{n-1}\left[-\frac{\delta_{\alpha, \beta}}{\left|x_{n}-x_{k}\right|^{s}}+s \frac{\left(x_{n}^{\alpha}-x_{k}^{\alpha}\right)\left(x_{n}^{\beta}-x_{k}^{\beta}\right)}{\left|x_{n}-x_{k}\right|^{s+2}}\right] .
$$

Further

$$
\begin{aligned}
U_{j, \alpha ; j, \beta}= & g e_{0}^{2} \sum_{k=1, k \neq j}^{n-1}\left[-\frac{\delta_{\alpha, \beta}}{\left|x_{j}-x_{k}\right|^{s}}+s \frac{\left(x_{j}^{\alpha}-x_{k}^{\alpha}\right)\left(x_{j}^{\beta}-x_{k}^{\beta}\right)}{\left|x_{j}-x_{k}\right|^{s+2}}\right] \\
& -e_{0} e_{n}^{0}\left[-\frac{\delta_{\alpha, \beta}}{\left|x_{j}-x_{n}\right|^{s}}+s \frac{\left(x_{j}^{\alpha}-x_{n}^{\alpha}\right)\left(x_{j}^{\beta}-x_{n}^{\beta}\right)}{\left|x_{j}-x_{n}\right|^{s+2}}\right] .
\end{aligned}
$$

As a result,

$$
\begin{aligned}
U_{j, \alpha ; j, \alpha}= & g e_{0}^{2} \sum_{k=1, k \neq j}^{n-1}\left[-\frac{1}{\left|x_{j}-x_{k}\right|^{s}}+s \frac{\left(x_{j}^{\alpha}-x_{k}^{\alpha}\right)^{2}}{\left|x_{j}-x_{k}\right|^{s+2}}\right] \\
& -e_{0} e_{n}^{0}\left[-\frac{1}{\left|x_{j}-x_{n}\right|^{s}}+s \frac{\left(x_{j}^{\alpha}-x_{n}^{\alpha}\right)^{2}}{\left|x_{j}-x_{n}\right|^{s+2}}\right], \\
\sum_{\alpha=1}^{d} U_{j, \alpha ; j, \alpha}= & g e_{0}^{2}(d-s)\left[-\sum_{k=1, k \neq j}^{n-1} \frac{1}{\left|x_{j}-x_{k}\right|^{s}}+e_{0} e_{n}^{0} \frac{1}{\left|x_{j}-x_{n}\right|^{s}}\right] .
\end{aligned}
$$

For the equilibrium, we have

$$
\begin{gathered}
\left|x_{j}-x_{n}\right|=a, \\
\left|x_{j}\right|=a, \quad x_{j}=a z^{j}, z=e^{i(2 \pi /(n-1))} \in \mathbb{C} .
\end{gathered}
$$

Then, one derives

$$
\sum_{\alpha=1}^{d} U_{j, \alpha ; j, \alpha}^{0}=\frac{g}{a^{s}}(s-d)\left[\sum_{k=1}^{n-2} e_{0}^{2} \frac{1}{\left|1-z^{k}\right|^{s}}-e_{0} e_{n}^{0}\right] .
$$

Besides, we have

$$
\begin{gathered}
U_{n, \alpha ; n, \alpha}=-g e_{0} e_{n}^{0} \sum_{k=1}^{n-1}\left[-\frac{1}{\left|x_{n}-x_{k}\right|^{s}}+s \frac{\left(x_{n}^{\alpha}-x_{k}^{\alpha}\right)^{2}}{\left|x_{n}-x_{k}\right|^{s+2}}\right], \\
\sum_{\alpha=1}^{d} U_{n, \alpha ; n, \alpha}=g e_{0} e_{n}^{0}(d-s) \sum_{k=1}^{n-1} \frac{1}{\left|x_{n}-x_{k}\right|^{s}}, \\
\sum_{\alpha=1}^{d} U_{n, \alpha ; n, \alpha}^{0}=\frac{e_{0} e_{n}^{0}}{a^{s}} g(d-s)(n-1) .
\end{gathered}
$$

This gives

$\operatorname{Tr} M^{-1} U^{0}=\frac{e_{0}^{2}}{a^{s}} g(d-s)\left[m^{\prime}\left(e_{0}^{-1} e_{n}^{0}-\sum_{k=1}^{n-2} \frac{1}{\left|1-z^{k}\right|^{s}}\right)+m_{n}^{-1}(n-1) e_{0}^{-1} e_{n}^{0}\right]$. 
Now, let us apply the equalities

$$
\begin{aligned}
\left|1-z^{k}\right|^{2}= & 1-2 \cos \frac{2 \pi k}{n-1}+\cos ^{2} \frac{2 \pi k}{n-1}+\sin ^{2} \frac{2 \pi k}{n-1} \\
= & 2\left(1-\cos \frac{2 \pi k}{n-1}\right), \sum_{k=1}^{n-2} \frac{1}{\left|1-z^{k}\right|^{s}} \\
= & 2^{-s / 2} \sum_{k=1}^{n-2}\left(1-\cos \frac{2 \pi k}{n-1}\right)^{-s / 2}, \cos ^{2} j=\frac{1}{2}(1+\cos 2 j), 1 \\
& \quad-\cos 2 j=2\left(1-\cos ^{2} j\right) \\
= & 2 \sin ^{2} j, \sum_{k=1}^{n-2}\left(1-\cos \frac{2 \pi k}{n-1}\right)^{-\frac{s}{2}}=2^{-\frac{s}{2}} \sum_{k=1}^{n-2} \sin ^{-s} \frac{\pi k}{n-1} .
\end{aligned}
$$

These equalities and (63) prove (10).

For $n=3, s=3, d=1$, we have $e_{n}^{0}=4^{-1} e_{0}, e_{3, s}=2^{-s}=2^{-2}$ $e_{3, s-2}$

$$
\operatorname{Tr}^{-1} U^{0}=-\frac{e_{0}^{2}}{a^{3}}\left[4^{-1}\left(m_{1}^{-1}+m_{2}^{-1}\right)+m_{3}^{-1}\right] .
$$

For $n=3, s=3, d=2$, we have

$$
\operatorname{Tr} M^{-1} U^{0}=-\frac{e_{0}^{2}}{2 a^{3}}\left[4^{-1}\left(m_{1}^{-1}+m_{2}^{-1}\right)+m_{3}^{-1}\right]
$$

The last two equalities are derived in [6] with the help of the found eigenvalues of $M^{-1} U^{0}$ (see also Theorem 12).

From $\sin \pi / 3=\sin 2 \pi / 3=\sqrt{ } 3 / 2$, it follows

$$
\begin{aligned}
e_{4, s} & =2^{-s+1}\left(\frac{2}{\sqrt{3}}\right)^{s}, \\
e_{4, s-2} & =3 e_{4, s} .
\end{aligned}
$$

$\sin \pi / 4=\sin 3 \pi / 4=1 / \sqrt{ } 2$ implies

$$
\begin{aligned}
e_{5, s} & =2^{-s}\left(1+2^{1+s / 2}\right)=2^{-s}+2^{1-s / 2}, \\
e_{5, s-2} & =2^{2-s}+2^{2-s / 2}>2 e_{5, s} .
\end{aligned}
$$

We also have

$$
\begin{aligned}
e_{3}^{0} & =2^{-s+1} e_{0}, \\
e_{3, s} & =2^{-s}, \\
n & =3 .
\end{aligned}
$$

Equation (10) gives

$$
\operatorname{Tr} M^{-1} U^{0}=\frac{e_{0}^{2}}{(2 a)^{s}} g(d-s)\left[m_{1}^{-1}+m_{2}^{-1}+4 m_{3}^{-1}\right]
$$

This equality follows also from Theorem 12.

\section{Eigenvalues of $M^{-1} U^{0}$ for Three Charges}

The simplest example of a Coulomb system with equilibrium is the $d$-dimensional system of the three-point charges $e_{1}=-e_{0}$, $e_{2}=-e_{0}, e_{3}=2^{1-s} e_{0}>0$ with masses $m_{1}, m_{2}, m_{3}$ and the potential energy in (2) for $n=3$. Its equilibrium $x^{0}$ is determined by $x_{1}^{01}=-a, x_{2}^{01}=a, x_{3}^{01}=0, x_{j}^{0 \alpha}=0, j=1,2,3,1<\alpha \leq d$.

The main result of this section is the proof of the following theorem.

Theorem 12. In the one-dimensional system, $M^{-1} U^{0}$ has the doubly degenerate zero eigenvalue and the eigenvalue $-\left(m_{1}^{-1}+m_{2}^{-1}+4 m_{3}^{-1}\right) g u^{\prime}, u^{\prime}=(s-1)\left(e_{0}^{2} /(2 a)^{s}\right)$. In the $d$ -dimensional systems, $d>1, M^{-1} U^{0}$ has the zero eigenvalue, which is $2 d$ times degenerate and the eigenvalues $\left(m_{1}^{-1}+m_{2}^{-1}+4 m_{3}^{-1}\right) g u^{\prime}, \quad\left(m_{1}^{-1}+m_{2}^{-1}+4 m_{3}^{-1}\right)(s-1)^{-1} g u^{\prime}$, the latter of which is $d-1$ times degenerate.

Proof. We find eigenvalues of $U^{0}$ at first for the onedimensional case. We have

$$
\frac{\partial}{\partial x_{j}} U\left(x_{(3)}\right)=-g e_{j} \sum_{k=1, k \neq j}^{3} e_{k} \frac{x_{j}-x_{k}}{\left|x_{j}-x_{k}\right|^{s}},
$$

that is

$$
\begin{aligned}
g^{-1} \frac{\partial}{\partial x_{1}} U\left(x_{(3)}\right) & =-e_{0}^{2} \frac{x_{1}-x_{2}}{\left|x_{1}-x_{2}\right|^{s}}+e_{0} e_{3} \frac{x_{1}-x_{3}}{\left|x_{1}-x_{3}\right|^{s}}, \\
g^{-1} \frac{\partial}{\partial x_{2}} U\left(x_{(3)}\right) & =-e_{0}^{2} \frac{x_{2}-x_{1}}{\left|x_{1}-x_{2}\right|^{s}}+e_{0} e_{3} \frac{x_{2}-x_{3}}{\left|x_{2}-x_{3}\right|^{s}}, \\
g^{-1} \frac{\partial}{\partial x_{3}} U\left(x_{(3)}\right) & =e_{0} e_{3}\left[\frac{x_{3}-x_{1}}{\left|x_{1}-x_{3}\right|^{s}}+\frac{x_{3}-x_{2}}{\left|x_{2}-x_{3}\right|^{s}}\right] .
\end{aligned}
$$

The equality $\left(\partial / \partial x_{3}\right) U\left(x_{(3)}\right)=0$ holds for $x_{1}=x_{1}^{0}=-a$, $x_{2}=x_{2}^{0}=a, x_{3}^{0}=0$. This configuration is an equilibrium. This follows also from the equalities $\left(\partial / \partial x_{j}\right) U\left(x_{(3)}\right)=0, j=1,2$.

The second derivatives of the potential energy are calculated as follows:

$$
\begin{aligned}
\frac{\partial U\left(x_{(3)}\right)}{\partial x_{j} \partial x_{k}} & =\frac{\partial U\left(x_{(3)}\right)}{\partial x_{k} \partial x_{j}}=-\left.g(s-1) e_{j} e_{k}\right|_{j}-\left.x_{k}\right|^{-s}, \quad k \neq j, \\
\frac{\partial^{2}}{\partial x_{j}^{2}} U\left(x_{(3)}\right) & =g(s-1) e_{j} \sum_{k=1, k \neq j}^{3} e_{k}\left|x_{j}-x_{k}\right|^{-s} .
\end{aligned}
$$

Hence, the second derivatives of the potential energy at the equilibrium $U_{j, k}^{0}$ are given by 


$$
\begin{gathered}
U_{1,2}^{0}=U_{2,1}^{0}=-g(s-1) \frac{e_{0}^{2}}{2^{s} a^{s}}=-g u^{\prime}, \\
U_{3,1}^{0}=U_{1,3}^{0}=U_{2,3}^{0}=U_{3,2}^{0}=2 g u^{\prime}, \\
U_{1,1}=U_{2,2}=-g u^{\prime}, U_{3,3}=-4 g u^{\prime} .
\end{gathered}
$$

That is

$$
U^{0}=-g u^{\prime}\left(\begin{array}{ccc}
1 & 1 & q \\
1 & 1 & q \\
q & q & q^{2}
\end{array}\right)=u^{\prime} U^{\prime}, \quad q=-2 .
$$

Let us put

$$
M_{0}^{\prime}(\lambda, q)=\left(\begin{array}{ccc}
k_{1}-\lambda & k_{1} & q k_{1} \\
k_{2} & k_{2}-\lambda & q k_{2} \\
q k_{3} & q k_{3} & q^{2} k_{3}-\lambda
\end{array}\right), \quad k_{j}=m_{j}^{-1} .
$$

Then, taking into consideration

$$
\begin{gathered}
M^{-1} U^{0}-\lambda I=-g u^{\prime} M_{0}{ }^{\prime}\left(-\frac{\lambda}{g u^{\prime}}, q\right), \\
-\operatorname{Det}\left(M^{-1} U^{0}-\lambda I\right)=g^{3} u^{\prime 3} \operatorname{Det} M_{0}^{\prime}\left(-\frac{\lambda}{g u^{\prime}}, q\right), \quad q=-2
\end{gathered}
$$

and making the expansion of the determinant in the elements of the first row of $M_{0}^{\prime}$, we obtain

$$
\begin{aligned}
\operatorname{Det} M_{0}^{\prime}(\lambda, q)= & \left(k_{1}-\lambda\right)\left[\left(k_{2}-\lambda\right)\left(q^{2} k_{3}-\lambda\right)-q^{2} k_{2} k_{3}\right] \\
& -k_{1}\left[k_{2}\left(q^{2} k_{3}-\lambda\right)-q^{2} k_{2} k_{3}\right] \\
& +q k_{1}\left[q k_{2} k_{3}-q k_{3}\left(k_{2}-\lambda\right)\right] \\
= & \left.\left(k_{1}-\lambda\right)\left[\lambda^{2}-\lambda\left(k_{2}+q^{2} k_{3}\right)\right]\right] \\
& +\lambda k_{1} k_{2}+\lambda q^{2} k_{1} k_{3} \\
= & \lambda\left[\left(k_{1}-\lambda\right)\left(\lambda-q^{2} k_{3}-k_{2}\right)+k_{1} k_{2}+q^{2} k_{1} k_{3}\right] .
\end{aligned}
$$

Hence,

$$
\begin{gathered}
\operatorname{Det} M_{0}^{\prime}(\lambda, q)=\lambda^{2}\left(-\lambda+k_{1}+k_{2}+q^{2} k_{3}\right), \\
\operatorname{Det}\left(M^{-1} U^{0}-\lambda I\right)=-\lambda^{2}\left[\lambda+\left(m_{1}^{-1}+m_{2}^{-1}+4 m_{3}^{-1}\right) g u^{\prime}\right] .
\end{gathered}
$$

The last formula proves the theorem for the onedimensional case.
Let us consider the two-dimensional case. For the first partial derivatives of the planar potential energy, we obtain

$$
\begin{aligned}
& \frac{\partial}{\partial x_{1}^{\alpha}} U\left(x_{(3)}\right)=g\left(-e_{0}^{2} \frac{x_{1}^{\alpha}-x_{2}^{\alpha}}{\left|x_{1}-x_{2}\right|^{s}}+e_{0} e_{3} \frac{x_{1}^{\alpha}-x_{3}^{\alpha}}{\left|x_{1}-x_{3}\right|^{s}}\right), \\
& \frac{\partial}{\partial x_{2}^{\alpha}} U\left(x_{(3)}\right)=g\left(-e_{0}^{2} \frac{x_{2}^{\alpha}-x_{1}^{\alpha}}{\left|x_{1}-x_{2}\right|^{s}}+e_{0} e_{3} \frac{x_{2}^{\alpha}-x_{3}^{\alpha}}{\left|x_{2}-x_{3}\right|^{s}}\right), \\
& \frac{\partial}{\partial x_{3}^{\alpha}} U\left(x_{(3)}\right)=g\left(e_{0} e_{3}\left[\frac{x_{3}^{\alpha}-x_{1}^{\alpha}}{\left|x_{1}-x_{3}\right|^{s}}+\frac{x_{3}^{\alpha}-x_{2}^{\alpha}}{\left|x_{2}-x_{3}\right|^{s}}\right]\right) .
\end{aligned}
$$

The last equality is zero at the equilibrium $-x_{1}^{1}=x_{2}^{1}$ $=a, x_{3}^{2}=x_{3}^{1}=x_{1}^{2}=x_{2}^{2}=0$. The first two give the equilibrium relation $e_{3}=2^{1-s} e_{0}$. by

The second derivatives of the potential energy are given

$$
\frac{\partial U\left(x_{(3)}\right)}{\partial x_{1}^{\alpha} \partial x_{2}^{\beta}}=\frac{\partial U\left(x_{(3)}\right)}{\partial x_{2}^{\beta} \partial x_{1}^{\alpha}}=g e_{0}^{2}\left[\frac{\delta_{\alpha, \beta}}{\left|x_{1}-x_{2}\right|^{s}}-s \frac{\left(x_{1}^{\alpha}-x_{2}^{\alpha}\right)\left(x_{1}^{\beta}-x_{2}^{\beta}\right)}{\left|x_{1}-x_{2}\right|^{s+2}}\right], \quad \alpha, \beta=1,2,
$$

$$
\begin{aligned}
\frac{\partial U\left(x_{(3)}\right)}{\partial x_{k}^{\alpha} \partial x_{3}^{\beta}}= & \frac{\partial U\left(x_{(3)}\right)}{\partial x_{3}^{\beta} \partial x_{k}^{\alpha}}=-g e_{0} e_{3}\left[\frac{\delta_{\alpha, \beta}}{\left|x_{k}-x_{3}\right|^{s}}-s \frac{\left(x_{k}^{\alpha}-x_{3}^{\alpha}\right)\left(x_{k}^{\beta}-x_{3}^{\beta}\right)}{\left|x_{k}-x_{3}\right|^{s+2}}\right], \quad k, \alpha, \beta=1,2, \\
\frac{\partial^{2} U\left(x_{(3)}\right)}{\partial x_{j}^{\beta} \partial x_{j}^{\alpha}}= & g e_{0}^{2}\left[-\frac{\delta_{\alpha, \beta}}{\left|x_{1}-x_{2}\right|^{s}}+s \frac{\left(x_{1}^{\alpha}-x_{2}^{\alpha}\right)\left(x_{1}^{\beta}-x_{2}^{\beta}\right)}{\left|x_{1}-x_{2}\right|^{s+2}}\right] \\
& +e_{0} e_{3}\left[\frac{\delta_{\alpha, \beta}}{\left|x_{j}-x_{3}\right|^{s}}-s \frac{\left(x_{j}^{\alpha}-x_{3}^{\alpha}\right)\left(x_{j}^{\beta}-x_{3}^{\beta}\right)}{\left|x_{j}-x_{3}\right|^{s+2}}\right], \quad j, \alpha, \beta=1,2,
\end{aligned}
$$

$$
\begin{aligned}
\frac{\partial^{2} U\left(x_{(3)}\right)}{\partial x_{3}^{\beta} \partial x_{3}^{\alpha}}= & g e_{0} e_{3}\left[\frac{\delta_{\alpha, \beta}}{\left|x_{1}-x_{3}\right|^{s}}-s \frac{\left(x_{1}^{\alpha}-x_{3}^{\alpha}\right)\left(x_{1}^{\beta}-x_{3}^{\beta}\right)}{\left|x_{1}-x_{3}\right|^{s+2}}\right. \\
& \left.+\frac{\delta_{\alpha, \beta}}{\left|x_{2}-x_{3}\right|^{s}}-s \frac{\left(x_{2}^{\alpha}-x_{3}^{\alpha}\right)\left(x_{2}^{\beta}-x_{3}^{\beta}\right)}{\left|x_{2}-x_{3}\right|^{s+2}}\right] .
\end{aligned}
$$

For the matrix of the second derivatives at the equilibrium, we derive

$$
\begin{aligned}
U_{1, \alpha ; 1, \beta}^{0}= & U_{2, \alpha ; 2, \beta}^{0}=g e_{0}^{2}\left[\delta_{\alpha, \beta}\left(-\frac{1}{(2 a)^{s}}+\frac{2^{1-s}}{a^{s}}\right)\right. \\
& \left.+s \delta_{\alpha, 1} \delta_{\beta, 1}\left(\frac{1}{(2 a)^{s}}-\frac{2^{1-s}}{a^{s}}\right)\right] \\
= & g \frac{e_{0}^{2}}{(2 a)^{s}} \delta_{\alpha, \beta}\left(1-s \delta_{\alpha, 1} \delta_{\beta, 1}\right)=4^{-1} U_{3, \alpha ; 3, \beta}^{0}, \\
U_{1, \alpha ; 2, \beta}^{0}= & U_{2, \beta ; 1, \alpha}^{0}=g \frac{e_{0}^{2}}{(2 a)^{s}} \delta_{\alpha, \beta}\left(1-s \delta_{\alpha, 1} \delta_{\beta, 1}\right), \\
U_{k, \alpha ; 3, \beta}^{0}= & U_{3, \beta ; k, \alpha}^{0}=-g \frac{2 e_{0}^{2}}{(2 a)^{s}} \delta_{\alpha, \beta}\left(1-s \delta_{\alpha, 1} \delta_{\beta, 1}\right), \quad k, \alpha, \beta=1,2 .
\end{aligned}
$$


Let us introduce the renumeration

$$
\begin{aligned}
& (1,1)=1, \\
& (2,1)=2, \\
& (3,1)=3, \\
& (1,2)=4, \\
& (2,2)=5, \\
& (3,2)=6, \\
& m_{4}=m_{1}, \\
& m_{5}=m_{2}, \\
& m_{6}=m_{3},
\end{aligned}
$$

where the first and second numbers in the round brackets correspond to the lower and upper indices of variables. Then, $U_{j, k}^{0}=U_{k, j}^{0}=0, j \leq 3, k \geq 4$ and

$U_{1,1}^{0}=U_{2,2}^{0}=4^{-1} U_{3,3}^{0}=-g u^{\prime}, U_{1,2}^{0}=-g u^{\prime}, U_{1,3}^{0}=U_{2,3}^{0}=2 g u^{\prime}, u^{\prime}=(s-1) \frac{e_{0}^{2}}{(2 a)^{s}}$,

$U_{4,4}^{0}=U_{5,5}^{0}=4^{-1} U_{6,6}^{0}=g c^{\prime}, U_{4,5}^{0}=g c^{\prime}, U_{4,6}^{0}=U_{5,6}^{0}=-2 g c^{\prime}, c^{\prime}=(s-1)^{-1} u^{\prime}$

This means

$$
U^{0}=u^{\prime} U^{\prime} \oplus-c^{\prime} U^{\prime}
$$

where $U^{\prime}$ is given by (75).

Let $M^{\prime \prime}=M^{\prime} \oplus M^{\prime}$ and $M^{\prime}$ be the $3 \times 3$ diagonal matrix with the elements $m_{1}, m_{2}, m_{3}$. Then,

$$
\begin{aligned}
& M^{\prime \prime-1} U^{0}-\lambda I=-g u^{\prime} M_{0}^{\prime}\left(-\frac{\lambda}{g u^{\prime}},-2\right) \oplus g c^{\prime} M^{\prime}{ }_{0}\left(\frac{\lambda}{g c^{\prime}},-2\right), \\
& \operatorname{Det}\left(M^{\prime^{\prime}}-1 U^{0}-\lambda I\right)=-u^{\prime 3} c^{\prime 3} \operatorname{Det} M_{0}^{\prime}\left(-\frac{\lambda}{g u^{\prime}},-2\right) \operatorname{Det} M_{0}^{\prime}\left(\frac{\lambda}{g c^{\prime}},-2\right) \text {. }
\end{aligned}
$$

From this equality and (79), we derive

$$
\begin{aligned}
-\operatorname{Det}\left(M^{-1} U^{0}-\lambda I\right)= & \lambda^{4}\left[-\lambda+\left(m_{1}^{-1}+m_{2}^{-1}+4 m_{3}^{-1}\right) g c^{\prime}\right] \\
& \cdot\left[\lambda+\left(m_{1}^{-1}+m_{2}^{-1}+4 m_{3}^{-1}\right) g u^{\prime}\right] .
\end{aligned}
$$

This concludes the proof for the two-dimensional case.

All the formulas concerning partial derivatives of the potential energy of this sections will be true if one extends the previous condition $\alpha, \beta=1,2$ to the condition $\alpha, \beta=1,2$ $, 3, \cdots, d$.
Let $d=3$ and let us continue the renumeration of the variables:

$$
\begin{aligned}
& (1,3)=7, \\
& (2,3)=8, \\
& (3,3)=9, \\
& m_{7}=m_{1}, \\
& m_{8}=m_{2}, \\
& m_{9}=m_{3} .
\end{aligned}
$$

It is not difficult to see that $U_{j, k}^{0}=U_{k, j}^{0}=0, j \leq 6, k \geq 7$ and $U_{7,7}^{0}=U_{8,8}^{0}=4^{-1} U_{9,9}^{0}=g c^{\prime}, U_{7,8}^{0}=g c^{\prime}, U_{7,9}^{0}=U_{8,9}^{0}=-2 g c^{\prime}$. Hence,

$$
U^{0}=U^{\prime \prime} \oplus-c^{\prime} U^{\prime}
$$

where $U^{\prime \prime}$ coincides with the planar $U^{0}$. Moreover,

$$
\begin{gathered}
=M^{\prime \prime} \oplus M^{\prime}=M^{\prime} \oplus M^{\prime} \oplus M^{\prime}=\left(\oplus M^{\prime}\right)^{3}, \\
M^{-1} U^{0}=M^{\prime \prime-1} U^{\prime \prime} \oplus-c^{\prime} M^{\prime-1} U^{\prime} .
\end{gathered}
$$

As a result,

$$
\begin{aligned}
-\operatorname{Det}\left(M^{-1} U^{0}-\lambda I\right)= & \lambda^{6}\left[-\lambda+\left(m_{1}^{-1}+m_{2}^{-1}+4 m_{3}^{-1}\right) g c^{\prime}\right]^{2} \\
& \cdot\left[\lambda+\left(m_{1}^{-1}+m_{2}^{-1}+4 m_{3}^{-1}\right) g u^{\prime}\right] .
\end{aligned}
$$

For the general case, we generalize the above renumeration as follows:

$$
\begin{gathered}
(1, d)=3(d-1)+1, \\
(2, d)=3(d-1)+2, \\
(3, d)=3(d-1)+3, \\
m_{3(d-1)+1}=m_{1}, \\
m_{3(d-1)+2}=m_{2}, \\
m_{3(d-1)+3}=m_{3} .
\end{gathered}
$$

It is not difficult to see that $U_{j, k}^{0}=U_{k, j}^{0}=0, j \leq 3(d-1), k$ $\geq 3(d-1)+1 \quad$ and $\quad U_{l+1, l+1}^{0}=U_{l+2, l+2}^{0}=4^{-1} U_{l+3, l+3}^{0}=g c^{\prime}$, $U_{l+1, l+2}^{0}=g c^{\prime}, U_{l+1, l+2}^{0}=U_{l+2, l+3}^{0}=-2 g c^{\prime}, l=3(d-1)$.

Let $M=\left(\oplus M^{\prime}\right)^{d}$, then these equalities imply

$$
U^{0}=u^{\prime} U^{\prime}\left(\oplus-c^{\prime} U^{\prime}\right)^{d-1}, M^{-1} U^{0}=u^{\prime} M^{\prime-1} U^{\prime}\left(\oplus-c^{\prime} M^{\prime-1} U^{\prime}\right)^{d-1},
$$




$$
\begin{aligned}
-\operatorname{Det}\left(M^{-1} U^{0}-\lambda I\right)= & \lambda^{2 d}\left[-\lambda+\left(m_{1}^{-1}+m_{2}^{-1}+4 m_{3}^{-1}\right) g c^{\prime}\right]^{d-1} \\
& \cdot\left[\lambda+\left(m_{1}^{-1}+m_{2}^{-1}+4 m_{3}^{-1}\right) g u^{\prime}\right] .
\end{aligned}
$$

Theorem 12 is proved.

Remark 13. Existence of global bounded solutions of nonsingular ordinary differential equation is well-known [10].

Remark 14. For the systems (1) and (2) with $d>1, n=3, s>1$ , the speed of the converges to the regular polygon equilibrium does not depend on $d$ since $M^{-1} U^{0}$ has only three eigenvalues for all $d>1$. The explicit dependence of $M^{-1} U^{0}$ on $d$, sis given by (70) for $s>1, n=3$. One can also calculate it without difficulty for $s>1, n=4,5$.

\section{Conclusion}

We showed that for the regular polygon equilibrium for (2) determined by Theorem 7 and $s=d$, the conclusion of Theorem 2 is true.

Our results that follow from Theorem 2 and Theorem 4 for Coulomb systems and $s=d$ are formulated in the following way.

Let $s=3, d=2 ; s=2, d=3$, and $n=3,4,5$. Let also $s=3$, $d=1, n=3$. Then, there exists an integer $p, 1 \leq p<n d$, a positive number $\lambda$, and a bounded at positive time solution $x(t)$ of the equation of motion (3) with $h_{c}=0$ depending on $p$ real parameters, which is a real analytic function in them in a neighborhood of the origin, such that $\left\|x-x^{0}\right\|_{\lambda}<\infty,\|\dot{x}\|_{\lambda}$ $<\infty$, where $x^{0}$ is a regular polygon equilibrium. This result for $s=3, d=1, n=3$ follows also from Theorem (1).

Theorem 12 shows that

(1) for the systems (1) and (2) with $d=1, n=3, s=2$, the conclusion of Theorem 2 is not true since $M^{-1} U^{0}$ does not have negative eigenvalues in $\mathbb{R}^{3}$

(2) for the systems (1) and (2) with $d>1, n=3, s>1$, the speed of the converges to the regular polygon equilibrium does not depend on $d$ since $M^{-1} U^{0}$ has only three eigenvalues for all $d>1$

\section{Data Availability}

There is no database source for this article.

\section{Conflicts of Interest}

The author declares that there is no conflict of interest regarding the publication of this paper.

\section{References}

[1] V. Vladimirov, Equations of Mathematical Physics, Series in Pure and applied mathematics, Moscow, 1967.

[2] S. Fleischer and A. Knauf, "Improbability of collisions in nbody systems,” 2018, https://arxiv.org/abs/1802,08564v1.
[3] W. Skrypnik, "On exact solutions of Coulomb equation of motion of planar charges," Journal of Geometry and Physics, vol. 98, p. 285, 2015.

[4] W. Skrypnik, "On regular polygon solutions of Coulomb equation of motion of $\mathrm{n}+2$ charges $\mathrm{n}$ of which are planar," Journal of Mathematical Physics, vol. 57, no. 4, article 042904, 2016.

[5] W. Skrypnik, "Quasi-exact Coulomb dynamics of $\mathrm{n}+1$ charges $\mathrm{n}-1$ of which are equal," Reports on Mathematical Physics, vol. 80, pp. 73-86, 2017.

[6] W. Skrypnik, "Mechanical systems with singular equilibria and Coulomb dynamics of three charges," Ukrainian Mathematical Journal, vol. 70, p. 519, 2018.

[7] C. Siegel and J. Moser, Lectures on Celestial Mechanics, Springer-Verlag, Berlin, Heidelberg, New-York, 1971.

[8] M. Bilogliadov, "Equilibria of the field generated by point charges," 2014, https://arxiv.org/abs/1404.7198v1.

[9] A. Gabrielov, D. Novikov, and B. Shapiro, "Mystery of point charges," Proceedings of the London Mathematical Society, vol. 95, p. 443, 2007.

[10] P. Hartman, Ordinary Differential Equations, Wiley and Sons, New York, London, Sydney, 1964. 